\title{
Sri Lanka:
}

¿un conflicto étnico?

Tamer Al Najjar Trujillo alnajjar@uji.es 


\section{Resumen}

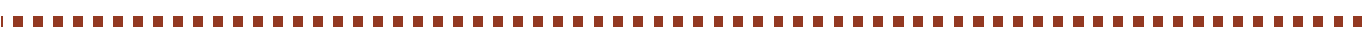

El presente trabajo tiene como objetivo analizar el conflicto armado de Sri Lanka para comprobar si se puede considerar un "conflicto étnico» o, por el contrario, las causas fundamentales de este son otras: la lucha por la autodeterminación de la minoría tamil y su discriminación durante años. Sin embargo, la cobertura (escasa) por parte de los medios de comunicación se ha limitado a explicar este conflicto como una lucha entre tamiles y cingaleses. Además, es necesario aclarar que el período que se va a analizar va desde 1983, cuando empieza la primera guerra, hasta 2009 cuando finaliza el conflicto armado con el asesinato del líder de los Tigres de Liberación Eelam Tamil por parte del ejército nacional

Así, se han consultado diversas fuentes a través de una amplia revisión bibliográfica: se hará referencia a datos e informes de la Escola de Cultura de Pau (para los procesos de paz), del Uppsala Conflict Data Program (número de fallecidos), del Instituto de Estudios Estratégicos (historia del conflicto) y de Human Rights Watch y Amnistía Internacional para analizar la situación de los Derechos Humanos en el país. Por otro lado, se hará mención a otros autores, como Vicenç Fisas, Camilla Orjuela o José María Tortosa, voces expertas en el tema de los conflictos armados y la construcción de la paz. Toda esta información recopilada se sintetiza en un cuadro análisis del conflicto diferenciando entre causas fundamentales, causas visibles, mecanismos, detonantes, factores que suavizan y aumentan la violencia, las formas de violencia y las consecuencias. De esta manera, no solo se analizará el conflicto de Sri Lanka desde un punto de vista histórico, como hasta ahora se ha venido estudiando. Todo ello permitirá extraer las conclusiones oportunas: cuáles son las causas reales de este conflicto armado.

Palabras clave: Sri Lanka, conflicto étnico, autodeterminación, cingaleses, tamiles.

\section{Introducción}

Durante 26 años la isla de Sri Lanka, en el océano Índico, ha estado sumida en una guerra que ha enfrentado al Gobierno contra el grupo insurgente Tigres de Liberación Eelam Tamil, que apostaba por la creación de un Estado independiente para la minoría tamil de las zonas septentrional y oriental del país. Y, aunque en la actualidad el conflicto armado ha finalizado, sus consecuencias todavía son visibles: miles de refugiados y desplazados, además de una fuerte polarización entre etnias. Es por ello que la elección de este conflicto 
no es baladí: pese a haber causado más de 70000 muertos (según la fuente varía el número) su cobertura mediática ha sido muy limitada comparada con la de otros conflictos también activos en el siglo XXI. Incluso algunos autores hablan de "guerra sin testigos» (Paz Vivas, 2013: 30). De esta manera, en primer lugar, se mostrará el cuadro con el análisis del conflicto para luego ser explicado de manera narrativa por apartados. También, y en la medida de lo posible, se pondrá rostro humano al conflicto para que las vivencias personales también puedan tener cabida en este trabajo y no caer en la deshumanización de los conflictos armados. De lo que se trata es, en definitiva, de ordenar y analizar la información existente de este conflicto, diferenciando causas reales (las que realmente pueden explicar el conflicto) de las causas visibles, aquellas que están en el entorno mediático y de los discursos, pero que no reflejan un análisis real ni exhaustivo de la realidad del conflicto que aquí se analiza. Por otro lado, también es importante visibilizar los diferentes procesos de paz fallidos, así como los actos de desobediencia civil llevados a cabo por la población tamil y cingalesa, ya que han sido olvidados y silenciados en los estudios tradiciones del conflicto de Sri Lanka.

\section{Objetivos}

Así pues, los objetivos que persigue el presente trabajo se pueden sintetizar de la siguiente manera:

1. Investigar las causas reales, fundamentales, del conflicto armado.

2. Estudiar si el conflicto armado se puede considerar como "étnico» o, por el contrario, hay otras razones profundas.

3. Establecer diferencias entre causas fundamentales, causas visibles, mecanismos, detonantes, factores que suavizan y aumentan la violencia, las formas de violencia y las consecuencias.

4. Extraer las conclusiones pertinentes sobre la realidad del conflicto armado.

\section{Material y método}

Para la consecución de los objetivos planteados es necesario seguir una correcta metodología que permita extraer los resultados pertinentes. Es por ello que para el análisis del conflicto armado de Sri Lanka se ha seguido el método de revisión bibliográfica (GómezLuna, 2014). De esta manera, se han seguido los siguientes pasos: definición del problema, búsqueda de la información, análisis de la información y organización de la información en cuadro análisis diferenciando entre causas fundamentales, causas visibles, 
mecanismos, detonantes, factores que suavizan y aumentan la violencia, las formas de violencia y las consecuencias.

Por lo tanto, partimos de la definición del problema: la información sobre el conflicto armado de Sri Lanka no diferencia entre causas fundamentales y causas visibles. De esta manera, se conseguirá tener un análisis exhaustivo del conflicto que se alejará de lo "aparente». Lo que se pretende es discernir la información de los resultados de los análisis históricos realizados sobre el conflicto de Sri Lanka. Así, no se trata únicamente de realizar una cronología del conflicto, sino de estudiar cada hecho concreto para incluirlo en los diferentes apartados aquí propuestos: causas fundamentales, causas visibles, mecanismos, detonantes, factores que suavizan y aumentan la violencia, las formas de violencia y las consecuencias. Así, pues, podremos extraer las pertinentes conclusiones del conflicto armado para saber cuáles han sido las razones profundas de este. Por todo lo anteriormente comentado, podemos considerar este trabajo como un artículo de revisión, ya que se han definido unos objetivos claros, se ha realizado una búsqueda bibliográfica, se ha organizado la información en un cuadro análisis (Guirao-Goris y otros, 2008: 5) para dar respuesta a una nueva pregunta: ¿es el conflicto de Sri Lanka un conflicto «étnico»?

\section{Resultados}

\subsection{Causas fundamentales}

Si tuviéramos que resumir las causas fundamentales de este conflicto armado serían dos: la discriminación de la población tamil, etnia minoritaria y de religión hinduista; y las demandas no satisfechas de autodeterminación de un Estado tamil independiente (Villellas, 2006: 3; Tortosa, 2000: 105; Fisas, 2010a: 133). Tras lograr Sri Lanka, antiguo Ceilán, la independencia de Reino Unido en 1948, los sentimientos etnocentristas empezaron a surgir (Mostefai, 2015). Durante la colonización británica se favoreció social y políticamente a la población tamil importada desde la India ${ }^{1}$ para trabajar en las plantaciones de té con el objetivo de calmar los movimientos antiimperialistas. Unos privilegios que llegaron a su fin con la llegada de un gobierno propio de mayoría cingalesa, etnia mayoritaria en el país y de religión budista (Villellas, 2006; Mostefai, 2015). Así, tras la llegada de la independencia estos tamiles occidentalizados fueron usados a modo de "chivo expiatorio" (Tortosa, 2000: 108) por su cercanía al gobierno colonial, «lo que llevó a que la mayoría cingalesa, tras la independencia, descargara sus frustraciones $y$ rencor en los tamiles privilegiados los cuales aglutinaron con la población tamil general» (Mostefai, 2015: 3). De esta manera,

\footnotetext{
${ }^{1}$ Procedentes del Estado Tamil Nadu en la India.
} 
comienza un proceso "cingalizador» (Villellas, 2006: 3) del país creando un Estado donde se empieza a excluir a la minoría tamil. La mutilación de derechos lingüísticos y religiosos, con el apoyo del clero budista, lleva consigo el comienzo de la creación de un nacionalismo tamil que se manifestó a través de demandas de autodeterminación de un Estado independiente durante los años sesenta y setenta (Mostefai, 2015: 3). Unas demandas que obtuvieron un no rotundo por parte del gobierno y que aumentaron la discriminación hacia la población tamil concentrada en las zonas norte y este del país (Villellas, 2006: 4). Se constata así el fracaso de un país federal multiétnico que había empezado a forjarse durante la última etapa colonizadora (Lewer y William, 2006: 231). Es por ello que este conflicto debe verse dentro de un contexto de reivindicación a través de las armas como última alternativa para no convertirse en ciudadanos de segunda, tras fracasar los intentos de desobediencia civil (Mostefai, 2015: 4). Por todo lo anteriormente dicho, y siguiendo a José María Tortosa, podemos entender el conflicto como una búsqueda efectiva de independencia del Estado del que forman parte (2000: 101). Vemos, así, que son varias las decisiones políticas las que nos hacen entender las causas del conflicto y no, como se comentará más tarde, las diferencias étnicas y religiosas de Sri Lanka (Rotberg, 1999: 5; Tortosa, 2000: 104). Sin embargo, todavía se sigue hablando de "conflicto étnico» (López, 2005).

\subsection{Causas visibles/aparentes}

Es cierto que podemos considerar a Sri Lanka como un país multiétnico y multicultural: el $75 \%$ de la población es cingalesa (de ellos, el $90 \%$ son budistas), el $18 \%$ son tamiles (de ellos, el $90 \%$ son hinduistas) y también hay presencia de musulmanes (7\%) y cristianos (Caro Bejarano, 2012: 369; López, 2005: 10-13; Central Intelligence Agency, 2016). Sin embargo, tal y como apunta el catedrático José María Tortosa, las diferencias culturales y étnicas «no son factores necesarios ni suficientes para el conflicto violento» (2000: 106). Esta teoría gana peso si tenemos en cuenta que históricamente la presencia de tamiles en el norte de la isla no supuso un peligro demográfico ni militar para sus vecinos, además de que la isla no estaba dividida en facciones tamiles y cingalesas antes de la guerra. Se trataría de una manera simplista y caricaturizada de explicar el conflicto desde el interior y el exterior (Mostafai, 2015: 7). Además, tal y como explica el profesor Seeni Mohamed Aliff, Sri Lanka ha sido históricamente famosa por la armonía entre religiones y etnias; una armonía que se fue deteriorando con la llegada de la independencia $^{2}$ (2015: 110-11). Los problemas comienzan cuando

\footnotetext{
${ }^{2}$ Traducción propia.
} 
esas diferencias se utilizan a modo de discriminación, como en el caso que nos ocupa: "Tiene una cierta lógica el que, una vez establecidas las diferencias y decidido el sistema de discriminación, se produzcan entre los discriminados diversas reacciones» (Tortosa, 2000: 107). Por otro lado, las diferencias religiosas tampoco explican las causas reales, ya que, aunque el clero budista haya apoyado ciertas medidas discriminatorias hacia los tamiles, esto responde a un interés de mantener el statu quo y como forma de mecanismo para polarizar grupos más que por motivos religiosos per se (Mostefai, 2015: 3; Villellas, 2006: 3).

\subsection{Mecanismos de movilización}

En primer lugar, los nacionalismos juegan un papel importante en la construcción de los grupos, en la configuración del yo-ellos. Así, los cingaleses patrióticos ven Sri Lanka como una isla sagrada donde se debe preservar el budismo. Del otro lado, los tamiles independentistas respondieron con una retórica basada en el chauvinismo, es decir, una exaltación de lo propio frente a lo extranjero (Rotberg, 1999: 8). Así comienzan las dicotomías entre cingaleses-tamiles y budistas-hinduistas. De esta manera, las diferencias étnicas se convierten en un mecanismo de movilización eficaz donde, incluso, la imagen de los "otros» se inventa parcialmente para cohesionar y reafirmar la pertenencia a un grupo (Tortosa, 2000: 107). Por otro lado, la construcción dentro del imaginario colectivo de pueblo elegido por parte del clero budista y la comunidad cingalesa es clave para entender la construcción del Estado postcolonial, de forma que sitúa a los tamiles como enemigo al que vencer (Villellas, 2006: 3). Una retórica amplificada por la utilización de ciertos textos sagrados por parte de budistas ultraconservadores para justificar la violencia contra los rebeldes tamiles (Vélez, 2004: 61). Por último, debemos entender el discurso de "los primeros pobladores» que viene a apoyar todas las retóricas anteriores: desde la esfera política se comienza a hablar de un sentimiento de pertenencia histórica por parte de los cingaleses, lo que viene a aumentar los sentimientos antitamiles (Mostefai, 2015: 7) y a crear la etiqueta de "forasteros» desde los dos bandos (Paz Vivas, 2015: 28).

\subsection{Detonantes/oportunidades}

Una vez llegada la independencia de Sri Lanka en 1948, se negó la ciudadanía a los trabajadores tamiles hindúes que llegaron desde Tamil Nadu (India) tras la aprobación de la Ley de Ciudadanía de Ceilán. Así, quedaron en una situación de expatriación que dio lugar al exilio forzoso de miles de ellos (Caro Bejarano, 2012: 379; Mostefai, 2015: 3). Junto a esto, la Constitución de 1972 establecía el 
cingalés y el budismo como lengua y religión oficiales. Se institucionaliza así la discriminación hacia la minoría tamil reforzada por la ley Solo Cingalés que favorecía a los cingaleses en la administración del país (Caro Bejarano, 2012: 371; Lewer y William, 2006: 231). Ante esto el partido Frente Unido de Liberación Tamil pide la autodeterminación de un propio Estado tamil, demanda apoyada por diferentes organizaciones tamiles a través de la Resolución Vaddukoddai en 1976. Ese mismo año se fundan los Tigres de Liberación Eelam Tamil por Velupilai Braphakaran, sucesores de los Nuevos Tigres de Tamil: una guerrilla que ya había atentado contra varios políticos tamiles procingaleses (Mostefai, 2015: 3). Se dan, así, los primeros signos de tensión entre ambas comunidades desde 1978 a 1981, año en el que se produce la quema de una biblioteca de Jaffna (capital cultural para los tamiles) por parte de policías y simpatizantes del Gobierno (Mostefai, 2015: 3). En 1983 los LTTE atacan una patrulla del ejército y causan la muerte de trece soldados. El Gobierno responde con fuertes represalias en las zonas de mayoría tamil en las que mueren entre 2000 y 3000 civiles (Caro Bejarano, 2012: 372). Comienza así una guerra cruenta. A partir de aquí empieza una escalada de la violencia que se ve incrementada por los asesinatos del primer ministro de India y del presidente de Sri Lanka por ataques suicidas vinculados a los LTTE (Crenshaw, 2007: 137; Pape, 2005: 15). A partir de este momento se dan otros dos acontecimientos importantes: el ataque por parte de los LTTE a dos botes patrulleros del ejército de Sri Lanka, que da comienzo a la tercera Guerra Eelam y los bombardeos por parte de la fuerza área del ejército nacional a varios campos de guerrilleros, que da comienzo a la cuarta Guerra Eelam. La ofensiva final en 2009, contra los reductos de los LTTE, que costó la vida de 20000 civiles (EI Mundo, 2009), pone fin a 26 años de conflicto tras la muerte de su fundador (Mostefai, 2015: 3-10). Por otro lado, como oportunidades, se puede destacar los Acuerdos de Thimpu (1985) con la India, ya que fue una oportunidad para dar salida al conflicto mediante el reconocimiento de una nación para las tamiles y aceptar su propia identidad. Sin embargo, El Gobierno de Sri Lanka rechazó estas ideas (Lewer y William, 2006: 233).

\subsection{Factores que suavizan la violencia}

Dentro de los factores que suavizan la violencia tenemos que mencionar el papel que han desempeñado ciertos actores internacionales como la India o Noruega. Así, es importante mencionar el acuerdo indio-esrilanqués de 1985. Aunque más bien existía un interés por poner fin al conflicto para que en la India las demandas de un Estado tamil en la zona de Tamil Nadu no tuvieran lugar, las fuerzas de manteamiento de la paz llegadas desde la India frenaron por un corto período de tiempo el conflicto armado (Lewer 
y William, 2006: 234-235). Por otro lado, en el año 2002, y gracias en parte a la mediación de países como Noruega, se llegó a un acuerdo de alto el fuego entre el Gobierno y los LTTE, rebajando las tensiones hasta 2007, aunque Noruega había abandonado el proceso de paz y los LTTE se habían retirado de las conversaciones (Villellas, 2006: 4; Lewer y William, 2006: 236-237). Aunque hubo muchos procesos de paz -todos fallidos-, todos tienen en común que han evitado numerosas muertes, han puesto de relieve la capacidad de diálogo entre los actores y han querido desvincular las diferencias étnicas del conflicto armado en sí (Villellas, 2006: 4). Además, los diferentes acuerdos se han preocupado por tener en cuenta el papel de las mujeres, principales víctimas: visibilizar la vulneración de sus derechos y mostrar un rol activo en las operaciones de mantenimiento de la paz (Villellas, 2006: 9; Consejo de Seguridad, 2000).

\subsection{Factores que agravan la violencia}

La intervención de la India en el conflicto se puede considerar como uno de los mayores agravantes. No solo dio apoyo logístico a los Tigres de Liberación Eelam Tamil durante los años ochenta, sino que, además, la impopularidad de las fuerzas de paz en las zonas este y norte de la isla llevó consigo la autorización por parte del Gobierno para dar armas a los LTTE para combatir a los militares hindúes (Mostefai, 2015: 4; Caro Bejarano, 2012: 382). Todo ello provocó el reforzamiento de los Tigres de Liberación y fue el comienzo de la internacionalización del conflicto que se vería amplificada por intervenciones extranjeras en los procesos de paz (Lewer y William, 2006: 234). De esta manera y, aunque los factores geopolíticos no son ejes motrices del conflicto, la intervención de China como proveedor de armas (Caro Bejarano, 2012: 372) y la participación de Noruega, EE. UU., la ONU y la UE, permitió a los LTTE argumentar la falta de imparcialidad y la excesiva internacionalización en los procesos de paz (Villellas, 2006: 6). Tal y como apunta Vicenç Fisas, lo aconsejable dentro de los procesos de paz es contar con pocos actores (Fisas, 2010b: 40). Tras calificar la UE y EEUU a los LTTE como grupo terrorista (Fisas, 2010b: 135), el Gobierno de Sri Lanka aprobó el Acta de Prevención del Terrorismo lo que supuso una intervención militar mayor (Caro Bejarano, 2012: 391) y aumentaron las dificultades para llegar a un proceso de paz (Villellas, 2006: 12). Este grupo armado, además, recibió el apoyo de la diáspora tamil desde los países con mayor número de refugiados tamiles, lo que supuso una legitimación de la violencia mayor para conseguir un Estado propio (Caro Bejarano, 2012: 367; Villellas, 2006: 8). Además, los ataques contra periodistas, las restricciones en las informaciones (Amnistía Internacional, 2008: 352; Reporteros Sin Fronteras, 2009: 180; Amnistía Internacional, 2009), la prohibición de 
la entrada de la ONU y ONG por parte del Gobierno desde 2008 (Caro Bejarano, 2012: 391) y las consecuencias del tsunami de 2004 agravaron aún más el conflicto: se invisibilizó el sufrimiento humano y se dejó desamparados a miles civiles que necesitaban ayuda humanitaria (Médicos Sin Fronteras, 2009). Por ello, este conflicto se puede considerar una «guerra sin testigos» (Paz Vivas, 2013: 30).

\subsection{Formas de violencia}

Las violaciones y abusos sexuales hacia la población tamil (mujeres, hombres, niños y niñas) por parte de las fuerzas armadas de Sri Lanka se convirtieron en un método sistemático y aceptado por el Gobierno para mostrar el dominio sobre las zonas norte y este de la isla; como un método de tortura para obtener información de civiles que supuestamente tenían alguna relación con los LTTE (Human Right Watch, 2013: 29), y para sembrar el terror entre la población tamil que en muchas ocasiones nada tenía que ver con el grupo armado (International Crisis Group, 2011: 26). En definitiva, una forma de "aprender la lección» (Human Right Watch, 2013). Unos abusos sexuales institucionalizados y que continuaron con total impunidad tras la finalización del conflicto armado (Sooka, 2014: 1420). Además, los LTTE llevaron a cabo más ataques suicidas que Hamás durante todo el conflicto armado (Pape, 2005: 82). Este grupo marxista-leninista se inspiró en los ataques de Hezbollah como método de presión hacia el Estado para conseguir un Estado tamil independiente (Pape, 2005: 82). Así, los Tigres Negros, como llamaban a los suicidas, llevaron a cabo 76 ataques hacia políticos, militares y civiles que costaron la vida de 906 personas (Pape, 2005: 95). Un método que tenía como objetivo terminar con la ocupación cingalesa en las zonas norte y este y que demostraba la capacidad de actuación del grupo armado, escenificada en el ataque suicida en el Banco Central de Colombo en 1996 (Crenshaw, 2007: 138). Una muestra más de que se trata de una guerra por la autodeterminación y no una lucha entre etnias. Además, los LTTE han sido acusados del reclutamiento forzoso de niños soldados (Mostafai, 2015: 9; Lewer y William, 2006: 237).

\subsection{Consecuencias}

Como en toda guerra la pérdida de vidas humanas es la consecuencia principal. Según el Uppsala Conflict Data Program, desde 1989 hasta 2015 más de 65000 personas perdieron la vida. Sin embargo, desde Naciones Unidas elevan el número de fallecidos hasta los 100000 (RTVE, 2009). Más allá de una discusión sobre el número de muertos a causa de la guerra, lo que podemos observar es la gran cantidad de vidas humanas que sufren a causa de la guerra. Una situación que se agrava si tenemos en cuenta el drama 
de los refugiados y desplazados internos. Aunque los datos son inciertos, en 2014 más de 90000 personas se encontraban desplazadas internamente en Sri Lanka (Escola de Cultura Pau, 2016: 34).

Y, aunque el número ha descendido respecto a 2004 (352 374), sigue siendo la población tamil la más afectada, ya que han querido buscar una vida mejor ante la discriminación y la violencia del ejército y los LTTE durante el conflicto (Kumar Acharya, 2007). Además, muchos de los desplazados son musulmanes que hablan tamil y que fueron expulsados por los LTTE en 1990 (Rodríguez, 2012: 88). El último informe estadístico del Alto Comisionado de las Naciones Unidas para los Refugiados ya no ofrece datos sobre refugiados esrilanqueses, pero se calcula que solo en la India hay alrededor de 10000 refugiados (Kumar Acharya, 2007: 108), la gran mayoría en los campos de Tamil Nadu. Francia, Reino Unido, Alemania y Canadá han sido otros de los países receptores de refugiados de Sri Lanka, justamente donde la diáspora tamil tiene más presencia (Vicenç Fisas, 2010a: 133). En 2014, 122000 personas seguían desplazadas (IDMC, 2015). A esto hay que añadirle que los que quisieron volver a sus hogares no pudieron hacerlo por la extensa presencia de minas, aunque ya se han eliminado 1,1 millones de ellas (Caro Bejarano, 2012: 378). No solo supone abandonar tu hogar con un alto coste y en embarcaciones improvisadas (Pedrera, 2016), sino que, además, las condiciones de los campos de refugiados siguen dejando mucho que desear: falta de libertad de movimiento y atención médica, existencia de dificultades para el acceso a la educación y el incumplimiento de los estándares sanitarios en la alimentación (Kumar Acharya, 2007: 108). Unas lamentables condiciones de vida que se pueden resumir en estas palabras de un refugiado en Tamil Nadu (Kumar Acharya, 2007: 119):

[...] You have a cow.... go and see; your cow must be living in a better place and must be eating good, but here our condition is far from your cow's condition.... If I tell you won't believe, the street dogs have more respect than us.

En 2015 la ONU instó al nuevo Gobierno a realizar las investigaciones oportunas sobre los posibles crímenes de guerra y de lesa humanidad cometidos por los dos bandos, ya que la impunidad sigue estando presente en el país (Amnistía Internacional, 2015; Kaleck, 2015). Tras la decisión del presidente Sirisena de eliminar la prohibición de viajar al norte de la isla, parece que estas investigaciones están avanzando. Así, se da una oportunidad para que las violaciones de DDHH que tuvieron lugar durante todo el conflicto salgan a la luz (Amnistía Internacional, 2017) y se pueda avanzar en la reparación de daños y generar justicia para las familias de fallecidos y desaparecidos. Sin embargo, en el plano social uno de 
los principales problemas después de la guerra sigue siendo la polarización étnica (Orjuela, 2003) a consecuencia de las narrativas de creación y confrontación de grupos, como ya se ha mencionado. Por ello, la reconciliación en comunidades étnicas es una tarea enorme ya que han estado enfrentadas bajo sentimientos de odio (Aliff, 2016: 10), donde los sentimientos antitamiles siguen presentes (Amnistía Internacional, 2017: 405) y donde la sociedad civil ha sido silenciada durante décadas (Orjuela, 2003: 199).

\section{Discusión y conclusiones}

A lo largo de este trabajo académico hemos podido demostrar que las causas profundas no se corresponden con las diferencias étnicas y religiosas que existen en Sri Lanka, como desde el exterior pudiera parecer, sino con las demandas de autodeterminación no satisfechas como respuesta a un sistema de discriminación hacia la población tamil. Por ello la calificación de "conflicto étnico» no se corresponde con la realidad del conflicto armado. Pese a esto, desde las altas instancias políticas y religiosas se han usado estas diferencias para mantener un sistema de discriminación y polarizar a la sociedad. Además, como en otros conflictos, vemos que se utilizan ciertos textos religiosos, sobre todo por budistas conservadores, para legitimar la violencia contra los LTTE y los tamiles en general.

Así, vemos que la religión se usa, de nuevo, como una excusa para mantener el statu quo y las posiciones de poder establecidas durante décadas. Ante esto la posible visualización del conflicto por los medios de comunicación se vio gravemente afectada por las injerencias gubernamentales con ataques constantes hacia los periodistas. Ello ha aumentado las dificultades para generar justicia hacia los familiares por la ausencia de testigos, pero también deja en el olvido el sufrimiento humano durante décadas de conflictos. Un sufrimiento humano que se pudo haber mitigado si los procesos de paz hubieran dado sus frutos, pero las percepciones entre las partes, la excesiva internacionalización y la fuerte polarización no permitieron que los acuerdos de paz llegaran a buen puerto. De esta manera, el drama de los refugiados y los desplazados ha ido en aumento, un tema complejo que sigue sin resolverse completamente. Por ello, es indispensable para Sri Lanka que existan movimientos de reconciliación entre comunidades étnicas y que los miles de desplazados puedan volver a sus hogares, muchos de ellos destruidos durante la guerra. Sin embargo, los nuevos sentimientos antimusulmanes promovidos desde ciertos sectores radicales budistas ponen el peligro la creación de una convivencia pacífica. Solo el establecimiento de la verdad, la justicia y la reparación puede hacer posible que Sri Lanka no vuelva a vivir una guerra cruenta. 


\section{Cuadro de análisis del conflicto}

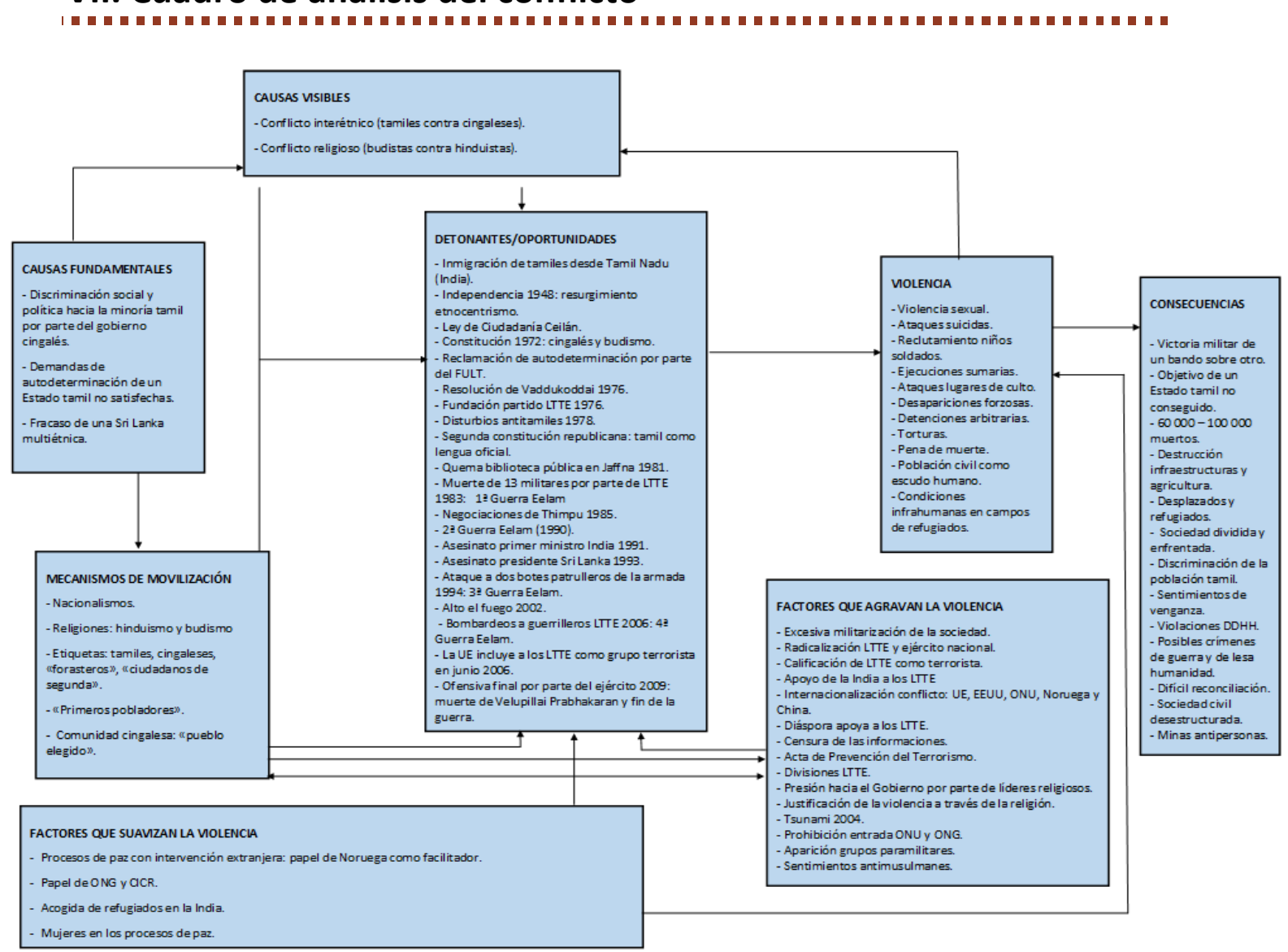

\section{Bibliografía}

Aliff, Seeni Mohamed. 2015. «Post-War Conflict in Sri Lanka: Violence against Sri Lankan Muslims and Buddhist Hegemony». International Letters of Social and Humanistic Sciences 59: 109125.

—. 2016. «Reconciliation in Post-war Sri Lanka». En 7th International Symposium 2017 (IntSym2017)- SEUSL - 07th \& 08th December 2017. University Park, Oluvil, Sri Lanka.

Amnistía Internacional. 2008. Informe 2008 Amnistía Internacional. La situación de los Derechos Humanos en el mundo. Madrid: Editorial Amnistía Internacional.

—. 2009. "Sri Lanka: Los ataques contra medios de comunicación libres ponen en peligro a la población civil desplazada». Amnistía Internacional Noticias. Acceso del 15 de abril de 2017. https://www.amnesty.org/es/latest/news/2009/08/sri-lankaattacks-free-media-put-displaced-civilians-risk-20090814/.

—. 2018. Informe 2016/17 Amnistía Internacional. La situación de los Derechos Humanos en el mundo. Madrid: Editorial Amnistía Internacional. 
Caro Bejarano, María José. 2012. «Sri Lanka». En Panorama

Geopolítico de los Conflictos 2012, editado por el Instituto Español de Estudios Estratégicos, 365-389. Madrid: Ministerio de Defensa, 366-389.

Central Intelligence Agency. 2016. "The World Factbook 2016». En www.cia.gov. Acceso del 12 de marzo de 2017. https://www.cia.gov/library/publications/the-worldfactbook/geos/ce.html.

Consejo de Seguridad. 2000. "Resolución 1325». Naciones Unidas. Acceso del 15 de abril de 2017. http://www.acnur.org/t3/fileadmin/Documentos/BDL/2006/175 9.pdf.

Crenshaw, Martha. 2007. "Explaining Suicide Terrorism: A Review Essay». Security Studies 16 (2): 133-162.

El Mundo. 2009. "Una masacre oculta: más de 20.000 civiles muertos en Sri Lanka». elmundo.es Acceso del 12 de abril de 2017. http://www.elmundo.es/elmundo/2009/05/29/internacional/12 43595576.html.

Escola de Cultura de Pau. 2016. Alerta 2016! Informe sobre conflictos, derechos humanos y construcción de paz. Barcelona: Icaria.

Fisas, Vicenç. 2010a. Anuario de procesos de paz 2010. Barcelona: Icaria Editorial/Escola de Cultura de Pau (UAB).

- 2010b. iAlto el fuego! Manual de procesos de paz. Barcelona: Icaria/Escola de Cultura de Pau (UAB).

Gómez Luna, Eduardo, Diego Fernando-Navas, Guillermo AponteMayo, y Luis Andrés Betancourt-Buitrago. 2014. «Metodología para la revisión bibliográfica y la gestión de información de temas científicos, a través de su estructuración y sistematización». Dyna 81 (184): 158-163.

Guirao-Goris, Josep Adolf, Angela Olmedo Salas y Esperanza Ferrer Ferrandis. 2008. «El artículo de revision». Revista Iberoamericana de Enfermería Comunitaria 1: 1-25.

Human Rights Watch. 2013. «We will you teach a lesson». Sexual Violence against Tamils by Sri Lankan Security Forces. Estados Unidos. Human Rights Watch.

International Crisis Group. 2011. «Sri Lanka: Women's Insecurity in the North and East». International Crisis Group. Acceso del 12 de abril de 2017. https://www.crisisgroup.org/asia/south-asia/srilanka/sri-lanka-women-s-insecurity-north-and-east. 
International Displacement Monitoring Centre. 2015. «Sri Lanka». International Displacement Monitoring Centre. Acceso del 15 de abril de $2017 . \quad$ http://www.internaldisplacement.org/database/country/?iso3=LKA.

Kaleck, Wolfgang. 2015. "Los criminales de guerra aún se sienten seguros en Sri Lanka». En eldiario.es. Acceso del 12 de abril de $2017 . \quad$ http://www.eldiario.es/contrapoder/Sri_Lankacrimenes_contra_la_humanidad-impunidad_6_413018701.html.

Kumar Acharya, Arun. 2007. "Ethnic Conflict and Refugees in Sri Lanka». Revista de Antropología Experimental 7: 107-121.

Lewer, Nick, y Joe William. 2006. «El proceso de paz en Sri Lanka: perspectivas históricas, políticas, económicas y de resolución del conflicto». En Anuario Asia-Pacífico 2004, coordinado por Oriol Farrés, 231-240. Barcelona: Masana Grafiquès.

López, G. 2005. El conflicto étnico de Sri Lanka. Madrid: Universidad Autónoma de Madrid.

Médicos Sin Fronteras. 2009. «Sri Lanka: 250.000 civiles atrapados sin acceso a ayuda humanitaria». Médicos Sin Fronteras. Acceso del 15 de abril de 2017. https://www.msf.es/actualidad/srilanka-250000-civiles-atrapados-acceso-ayuda-humanitaria.

Mostefai, Ahmed Fawzi. 2015. "Sri Lanka: Antes y Después de la Guerra», Observatorio Virtual Asia Pacífico. Acceso del 3 de marzo de 2017. http://www.utadeo.edu.co/files/collections/documents/field_at tached_file/sri-lanka-6-anos-despues-de-la-guerra.pdf.

Navarro, Fernando. 2008. "Un tsunami armado devorada Sri Lanka». El País. Acceso del 1 de marzo de 2017. http://internacional.elpais.com/internacional/2008/04/24/actua lidad/1208988009_850215.html.

Orjuela, Camilla. 2003. «Building Peace in Sri Lanka: A Role for Civil Society?». Journal of Peace Research 40 (2): 195-202.

Pape, Robert. 2005. Dying to win. The Strategic Logic of Suicide Terrorism. Nueva York: Ramdom House.

Paz Vivas, Luz María. 2013. "Sri Lanka. Historia de un conflicto». Acción Cultural Cristiana. Acceso del 25 de febrero de 2017. http://www.accionculturalcristiana.org/html/revista/r75/75sril.p df.

Perera, Suvendrini. 2016. Survival Media: The Politics and Poetics of Mobility and the War in Sri Lanka. Nueva York: Palgrave Macmillan. 
Radio Televisión Española. 2009. «Los 26 años de guerra en Sri Lanka se han cobrado 100.000 muertos según Naciones Unidas». rtve.es. Acceso del 15 de abril de 2017. http://www.rtve.es/noticias/20090520/26-anos-guerra-srilanka-se-han-cobrado-100000-muertos-segun-nacionesunidas/277431.shtml.

Reporteros Sin Fronteras 2009. La Libertad de Prensa en el Mundo 2009. Madrid: Reporteros Sin Fronteras.

Rodríguez Azcárate, Diego. 2012. Sri Lanka. Informe general. Madrid: Comisión Española de Ayuda al Refugiado. Acceso del 12 de abril de 2017. https://cear.es/wp-content/uploads/2013/08/SRILANKA.-2012.-Informe-general.pdf.

Rotberg, Robert. 1999. Creating Peace in Sri Lanka. Civil War and Reconciliation. Washington D.C.: Bookings Institution Press.

Sarjoon, Athambawa, Mohammad Agus Yusoff y Nordin Hussin. 2016. «Anti-Muslim Sentiments and Violence: A Major Threat to Ethnic Reconciliation and Ethnic Harmony in Post-War Sri Lanka». Religions 7 (10). doi:

https://doi.org/10.3390/rel7100125.

Sooka, Yasmin. 2014. An Unfinished War: Torture and Sexual Violence in Sri Lanka 2009-2014. Johannesburg: Foundation for Human Rights. Acceso del 14 de abril de 2017. http://www.stoptorture.com/.

Tortosa Blasco, José María. 2000. «Guerras por la identidad: de la diferencia a la violencia». Anuario CIP 2000: Globalización y Sistema Internacional, editado por Mariano Aguirre, 99-112. Barcelona: Icaria.

Uppsala Conflict Data Program. 2016. "Sri Lanka». Uppsala Conflict Data Program. Acceso del 14 de abril de 2017. http://ucdp.uu.se/\#country/780.

Vélez de Cea, J. Abraham. 2004. "Paz y violencia en el budismo». En 10 palabras clave sobre paz y violencia en las religiones, dirigido por Juan José Tamayo Acosta, 47-75. Navarra: Verbo Divino.

Villellas Ariño, María. 2006. "Sri Lanka: ¿una oportunidad definitiva para la paz?». Escola de Cultura de Pau. Acceso del 5 de marzo de 2017. http://escolapau.uab.cat/img/programas/alerta/articulos/06arti culo026.pdf. 